\title{
Novel transgenic mice with Cre-dependent co-expression of GFP and human ACE2: a safe tool for study of COVID- 19 pathogenesis
}

\author{
Alexandra V. Bruter • Diana S. Korshunova • Marina V. Kubekina • \\ Petr V. Sergiev • Anastasiia A. Kalinina - Leonid A. Ilchuk • Yuliya Yu. Silaeva • \\ Eugenii N. Korshunov $\cdot$ Vladislav O. Soldatov $(D)$ Alexey V. Deykin
}

Received: 12 January 2021 / Accepted: 26 March 2021 / Published online: 14 April 2021

(C) The Author(s), under exclusive licence to Springer Nature Switzerland AG 2021

\begin{abstract}
The current coronavirus disease (COVID19) pandemic remains one of the most serious public health problems. Increasing evidence shows that infection by severe acute respiratory syndrome coronavirus-2 (SARS-CoV-2) causes a very complex and multifaceted disease that requires detailed study. Nevertheless, experimental research on COVID-19 remains challenging due to the lack of appropriate animal models. Herein, we report novel humanized mice with Cre-dependent expression of hACE2, the main entry receptor of SARS-CoV-2. These mice carry $h A C E 2$ and GFP transgenes floxed by the STOP cassette, allowing them to be used as breeders for the creation of animals with tissue-specific coexpression of hACE2 and GFP. Moreover, inducible expression
\end{abstract}

AlexandraV. Bruter, DianaS. Korshunova and MarinaV. Kubekina have contributed equally to this work

A. V. Bruter · M. V. Kubekina · L. A. Ilchuk ·

Y. Yu. Silaeva · E. N. Korshunov · V. O. Soldatov $(\bowtie)$

Core Facility Centre, Institute of Gene Biology, Russian

Academy of Sciences, Vavilova st. 34/5, Moscow,

Russian Federation 119334

e-mail: korshunov@genebiology.ru

A. V. Bruter

e-mail: bruter@genebiology.ru

M. V. Kubekina

e-mail: kubekina@genebiology.ru

L. A. Ilchuk

e-mail: lechuk12@gmail.com of hACE2 makes this line biosafe, whereas coexpression with GFP simplifies the detection of transgeneexpressing cells. In our study, we tested our line by crossing with Ubi-Cre mice, characterized by tamoxifen-dependent ubiquitous activation of Cre recombinase. After tamoxifen administration, the copy number of the STOP cassette was decreased, and the offspring expressed $h A C E 2$ and GFP, confirming the efficiency of our system. We believe that our model can be a useful tool for studying COVID-19 pathogenesis because the selective expression of hACE2 can shed light on the roles of different tissues in SARS-CoV-2-associated complications. Obviously, it can also be used for preclinical trials of antiviral drugs and new vaccines.

\section{Graphic abstract}

Y. Yu. Silaeva

e-mail: silaeva@genebiology.ru

E. N. Korshunov

e-mail: pharmsoldatov@gmail.com

D. S. Korshunova $\cdot$ A. V. Deykin

Center for Precision Genome Editing and Genetic

Technologies for Biomedicine, Institute of Gene Biology,

Russian Academy of Sciences, Vavilova st. 34/5,

Moscow, Russian Federation 119334

e-mail: korshunova@genebiology.ru

A. V. Deykin

e-mail: deikin@igb.ac.ru 

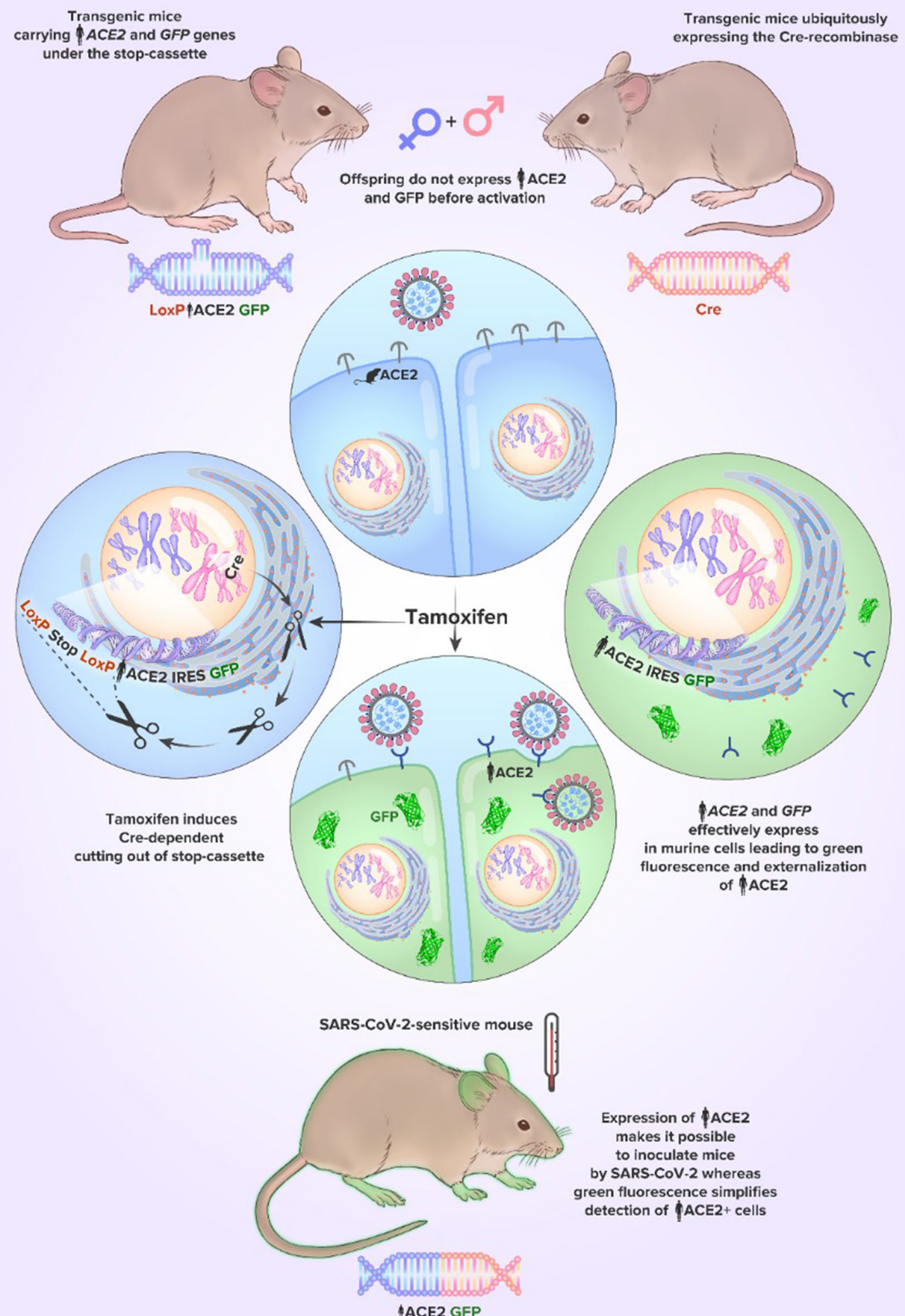

Expression of ACE2 makes it possible

to inoculate mice by SARS-CoV-2 whereas green fluorescence simplifies detection of AACE2+ cells 
Keywords COVID-19 - ACE2 - Transgenes · SARS-CoV-2 - Tamoxifen · Cre-recombinase

\section{Introduction}

Coronavirus disease (COVID-19) caused by severe acute respiratory syndrome coronavirus 2 (SARS$\mathrm{CoV}$-2) remains one of the most critical challenges for the medical and scientific communities. Since the first cases of infected patients in Wuhan (Zhu et al., 2020), our understanding of COVID-19 pathogenesis has been repeatedly transformed by the results of clinical and molecular research. Most data were obtained from clinical observational studies, such as cohort and casecontrol studies. In silico (Shang et al. 2020; Meirson et al. 2020) and in vitro (Hoffmann et al. 2020; Suzuki 2020) research also clarified the mechanisms of interactions between the virus and human cells. Nevertheless, opportunities to perform experiments to reveal the details of COVID-19 pathogenesis are limited by the absence of available animal test systems.

Recently, SARS-CoV-2 was shown to effectively replicate in several species, including nonhuman primates, ferrets, dogs, and cats (Lakdawala et al. 2020). Macaques infected with the novel virus were used for testing an inactivated vaccine (Gao et al. 2020). A study in ferrets revealed unexpected details regarding the anti-SARS-CoV-2 immune response (Blanco-Melo et al. 2020). However, there are unfortunately no naturally SARS-CoV-2-sensitive species

\footnotetext{
P. V. Sergiev

Institute of Functional Genomics, Lomonosov Moscow State University, Moscow, Russian Federation 119991

e-mail: petya@belozersky.msu.ru
}

among the routinely used laboratory animals. Obviously, the most convenient, available, and popular laboratory test system requiring no special housing conditions is mice. As wild-type mice support transient coronavirus infection without clinical signs of the disease (Subbarao et al. 2004), the use of genetically modified strains expressing human angiotensin-converting enzyme 2 (hACE2) (McCray et al. 2007; Tseng et al. 2007; Menachery et al. 2016; Yang et al. 2007; Bao et al. 2020; Soldatov et al. 2020; Masemann et al. 2020), the entry receptor for SARSCoV-2 (Lu et al. 2020), was proposed. Another proposed option was to deliver exogenous hACE2 to the alveolar epithelium via intranasal administration of replication-deficient adenovirus Ad5-hACE2 in wild-type mice (Sun et al. 2020; Hassan et al. 2020). Moreover, SARS-CoV-2 itself was genetically modified to effectively interact with murine ACE2 receptors, making any mouse suitable for COVID-19 studies without humanization (Dinnon et al. 2020; $\mathrm{Gu}$ et al. 2020).

Here, we report a novel SARS-CoV-2-sensitive transgenic mouse strain, Tg CAGLoxPStopACE2GFP (hereinafter referred to as hACE2(LoxP-Stop)), carrying $h A C E 2$ and green fluorescent protein $(G F P)$ transgenes under the floxed STOP cassette. The basic hallmark of this strain is the possibility of temporal and spatial control of transgene activation, allowing adjustment of the $h A C E 2$ gene expression profile by breeding with different Cre strains. In our study, we crossed hACE2(LoxP-Stop) mice with Ubi-Cre/ERT2 mice, in which Cre/ERT2 recombinase is expressed ubiquitously but is able to excise the STOP cassette only after administration of tamoxifen (Cre/ERT2 needs tamoxifen to be translocated into the nucleus). Their offspring successfully expressed hACE2 and GFP after tamoxifen induction, proving that the system works.

\section{Materials and methods}

Design and manufacturing of the transgene DNA construct

The DNA construct consisted of two basic parts: the pKB1 vector and the hACE2 ORF. pKB1, an ampicillin-resistant vector, was constructed for cloning genes intended for Cre-dependent expression. It

\section{O. Soldatov · A. V. Deykin}

Laboratory of Genome Editing for Veterinary and Biomedicine, Belgorod State National Research University, 85, Pobedy St., Belgorod,

Belgorod region, Russian Federation 308015 
contains insulators and terminators to protect the transgene from the position effect (Miyazaki et al. 1989; Chung et al. 1993; Deykin et al. 2019), the transcriptional unit under the control of the CAG promoter, and the STOP cassette (Lakso et al. 1992). The transcriptional unit consisted of multiple cloning sites for cloning of the transgene, the IRES element of the encephalomyocarditis virus in frame with the GFP gene and the SV40polyA signal. The pKB1 vector was constructed on the basis of the NIF vector (Zvartsev et al. 2019): we inserted a multiple cloning site (AgeI, EcoRV, MluI) between the STOP cassette and the IRES. Transgene expression in $\mathrm{pKB} 1$ is supposed to be activated only after excision of the STOP cassette by Cre recombinase.

Total RNA for $h A C E 2$ cloning was extracted from human kidney biopsy material. The tissue was homogenized with a Precellys 24 homogenizer, and RNA was extracted with the QIAGEN RNeasy Mini Kit. cDNA was synthesized with reverse transcriptase Superscript II (Invitrogen) and $\mathrm{d}(\mathrm{T}) 16$ oligo. The hACE2 gene (2400 bp) was amplified with Q5 polymerase (NEB) using primers P15 and P16 (all primer sequences are presented in the Appendix Table 1). The amplified fragment was digested with restriction enzymes BshTI and MluI (Thermo Fisher Scientific) and inserted into the pKB1 vector digested with the same enzymes (Fig. 1).

The circular plasmid DNA was then digested with PvuI and gel purified; a $13.5 \mathrm{kbp}$ linear fragment without the bacterial propagation elements was extracted with the QIAquick Gel Extraction Kit (Qiagen) and dissolved in TE buffer (Zvezdova et al. 2010) at a concentration of $1 \mathrm{ng} / \mu \mathrm{l}$.

Animals, pronuclear microinjections, and embryo transfer

To generate primary transgenic animals, we used (CBAxC57BL/6)F1 zygotes obtained from 3-weekold CBA and C57BL/6 breeders (Stolbovaya breeding station, Russian Federation). The transgene DNA construct was introduced by pronuclear microinjections as described earlier (Zvezdova et al. 2010). Surviving zygotes were cultivated in M16 medium at $37{ }^{\circ} \mathrm{C}$ and $6 \% \mathrm{CO}_{2}$ for $24 \mathrm{~h}$ and then transplanted into the oviducts of foster mothers at the 2-cell stage as previously described (Silaeva et al. 2018).
The B6. Cg-Ndor1Tg(UBC-cre/ERT2)1Ejb/1 J, also known as Ubi-Cre, mouse strain was purchased from the Jackson Laboratory (Goodwin et al. 2019). In this strain, chimeric Cre recombinase is expressed under the control of the UBC promoter. Chimeric Cre recombinase consists of a Cre recombinase domain and a domain representing a mutant form of the estrogen receptor, which lacks the ability to bind natural $17 \beta$-estradiol and binds only synthetic tamoxifen. Without tamoxifen, the protein is restricted to the cytoplasm, and only in the presence of tamoxifen translocates to the nucleus and performs recombination. Ubi-Cre mice can be maintained only in hemizygotes.

Animals were kept in the IGB RAN vivarium with artificial lighting (12 $\mathrm{h} / 12 \mathrm{~h}$ mode) at a temperature of $21-23{ }^{\circ} \mathrm{C}$ and humidity of $38-50 \%$; mice had free access to food and water.

\section{Tamoxifen treatment}

Tamoxifen (Hexal) was administered by gavage at a dose of $1 \mathrm{mg} / 100 \mathrm{~g}$ once a day for 20 days (2\% suspension formulations).

\section{Genotyping}

DNA samples were extracted from tail tissues samples. The first step of F0 genotyping was performed using the primer pair $\mathrm{P} 1$ and $\mathrm{P} 2$, specific to the $3^{\prime}$ region of the STOP cassette and the $5^{\prime}$-region of IRES, respectively (the 3000 bp PCR product band, Fig. 2). Advanced genotyping of F0 combined detection of the STOP cassette (P13, P14), the hACE2 ORF (P15, $\mathrm{P} 16)$, eGFP (P21, P22), and the terminator (P23, P24).

$\mathrm{F} 1$ genotyping was performed by detection of $h A C E 2$ and $e G F P$ ORF presence as described above and Ubi-Cre presence according to the protocol recommended by the Jackson Laboratory using P17, P18, P19, and P20 primers. All Ubi-Cre probes had a $324 \mathrm{bp}$ band as the internal positive control, and transgenic Ubi-Cre probes had a 475 bp band.

\section{Copy number measurement}

The transgene copy number was determined using logarithmic interpolation based on $C_{t}$ for the transgene and reference genes with known copy numbers. We chose murine Hprt (1 copy per genome in males, 2 in 


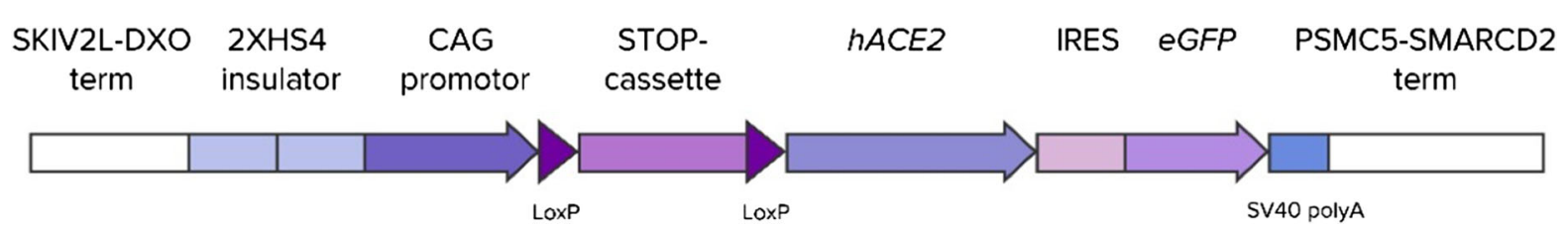

Fig. 1 pKB1-hACE2 linear construct intended for transgenesis

females, primers P5 and P6), Hbal (4 copies per genome, primers $\mathrm{P} 7$ and $\mathrm{P} 8$ ), and $H 3 c 7$ (18 copies per genome, primers $\mathrm{P} 9$ and P10) genes as the reference for comparison. For qPCR, we used SYBR Green mix with HS Taq polymerase (Evrogen, Russia). We used primers $\mathrm{P} 3$ and $\mathrm{P} 4$ for hACE2 copy number measurement. In experiments assessing the Cre recombinase effectivity, we also measured the STOP cassette copy number with primers $\mathrm{P} 13$ and P14.

\section{qPCR and gene expression measurement}

Blood for evaluation of hACE2 expression was collected from the retro-orbital venous sinus under anesthesia induced by Zoletil (2 mg/100 g) + Xylazin $(2 \mathrm{mg} / 100 \mathrm{~g})$. One hundred microliters of blood samples were mixed with $1 \mathrm{ml}$ of ammonium chloride-potassium $\mathrm{RBC}$ lysis buffer, incubated for $5 \mathrm{~min}$ at $25{ }^{\circ} \mathrm{C}$ and centrifuged for $5^{\prime}$ at $300 \mathrm{~g}$ at room temperature. The observed cell pellet was washed with PBS twice and used for downstream applications.

gDNA-free total RNA was extracted from blood cells with the RNeasy Plus Mini Kit (Qiagen). cDNA was synthesized with iScript ${ }^{\mathrm{TM}}$ Reverse Transcription Supermix (Bio-Rad), and real-time PCR was performed using SYBR GREEN mix (Evrogen) and primers P3, P4, P11, and P12. Real-time PCR with extracted RNA instead of cDNA was performed to prove the absence of genomic DNA contamination. The results were analyzed with CFX Manager Software (Bio-Rad).

\section{Western blot}

Peripheral blood mononuclear cells (PBMCs) of F1 mouse hACE2 (LoxP-Cre) were extracted as previously described (Silaeva et al. 2013). After RBC lysis, PBMCs were lysed in $200 \mu \mathrm{l}$ of RIPA buffer $(1 \mathrm{ml}$ RIPA: $150 \mathrm{mM}$ sodium chloride, $1.0 \%$ NP-40, $0.5 \%$ sodium deoxycholate, $0.1 \%$ SDS (sodium dodecyl sulfate), 50 mM Tris, pH 8.0; $2 \mu$ l PIC, $20 \mu$ PMSF).
The protein concentration was measured by the Bradford assay, and a calibration curve was built using BSA serial dilutions. The samples $(80 \mathrm{mg}$ of protein each) were loaded into $8 \%$ PAAG. Electrophoresis was performed in SDS buffer at $120 \mathrm{~V}$ for $1.5 \mathrm{~h}$ and the proteins were then transferred to a nitrocellulose membrane $(0.2 \mu \mathrm{m})$ at $250 \mathrm{~mA}$ for $1 \mathrm{~h}$ in transfer buffer with $20 \% \mathrm{MeOH}$. After the transfer, the membrane was incubated in 5\% nonfat dried milk dissolved in TBS. The primary anti-hACE2 antibody PAB886Hu01 (Cloud-Clone Corp., USA-China) was diluted 1:1500 in 1\% BSA in TBS buffer, and the membrane was stained for $18 \mathrm{~h}$ at $4{ }^{\circ} \mathrm{C}$. The membrane was then washed in TBS buffer and incubated with secondary anti-rabbit antibodies (anti-rabbit IgG, HRP-linked antibody \# 7074, Cell Signaling) diluted 1:1000 in 5\% nonfat dried milk dissolved in TBS for $1 \mathrm{~h}$. After washing in TBS buffer, chemiluminescent labels were activated with ECL and visualized in iBright.

\section{FACS analysis}

Extracted PBMCs were preincubated with Fc block (clone 2.4G2, BD Pharmingen, Franklin Lakes, NJ) $\left(10 \mathrm{~min}, 4{ }^{\circ} \mathrm{C}\right)$, incubated with polyclonal antihACE2 antibodies (Cloud-clone Corp., Product No. PAB886Hu01) $\left(40 \mathrm{~min}, 4^{\circ} \mathrm{C}\right)$ and then stained with secondary fluorescently labeled antibodies (Invitrogen, Catalog \# A-11010) (40 min, $4{ }^{\circ} \mathrm{C}$ ). The analysis was performed on a BD FACSCanto II flow cytometer (BD Bioscience) using FACSDiva 6.0 software (BD Bioscience). Dead cells were excluded from the analysis based on the parameters of the forward and side scatter and staining with propidium iodide (BD Bioscience). At least $10^{4}$ events per sample were collected to characterize the peripheral leukocyte populations. Data were processed using FlowJo 7.6 software (TreeStar Inc., Ashland, USA). 


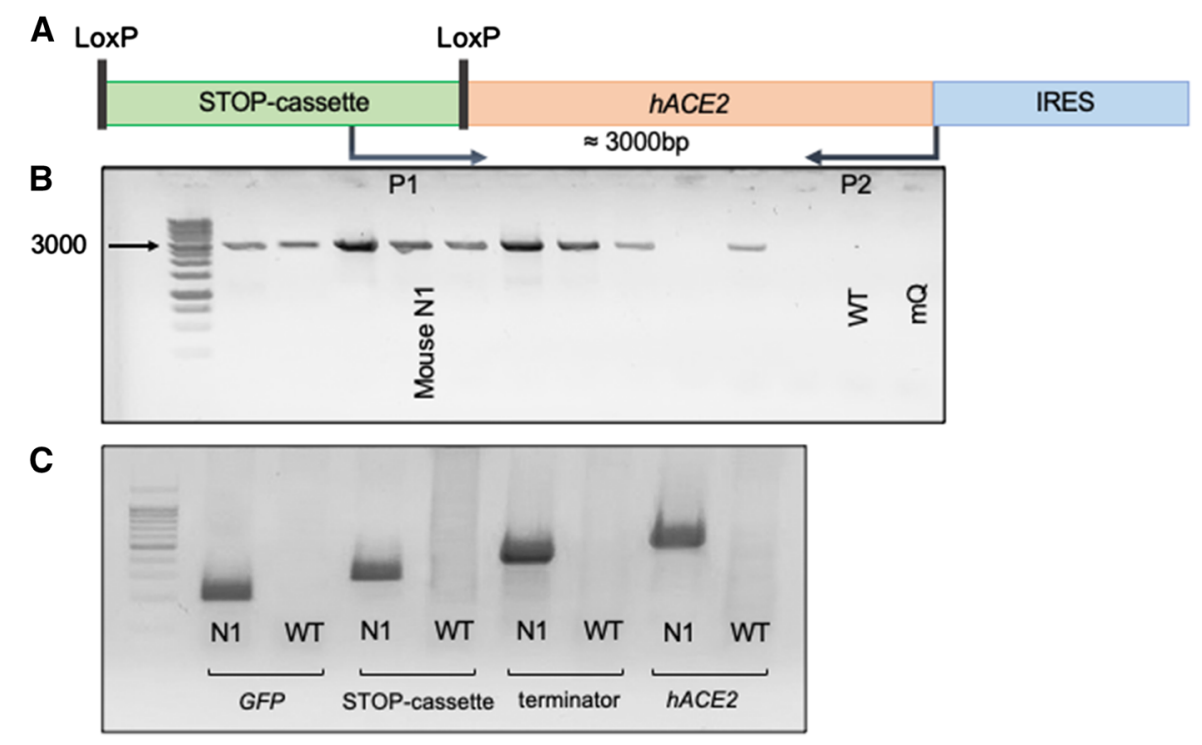

Fig. 2 F0 genotyping. a Primer positioning for primary genotyping; b F0 primary genotyping. Mouse N1 involved in further experiments is indicated; c Advanced genotyping of

\section{Statistical analysis}

Statistical processing was not applied due to the limited number of animals (only one in each group).

\section{Results}

Generation of primary transgenic mice

A total of 1511 zygotes were microinjected and transplanted into 138 recipients. Twenty-four recipients gave birth to 48 pups (F0). Nine pups carried the transgene, and 4 of them gave $\mathrm{F} 1$ offspring.

Being aware of mosaic patterns of transgene insertion, we nevertheless evaluated the transgene copy number in the F0 generation. Seven of 9 transgenes had copy numbers, significantly exceeding 1 -in the range between 4 and 27 copies per genome. This result indicates a high probability of multimers of the construct being inserted into the genome (Chen et al. 2001; Smirnov et al. 2020). Moreover, it is possible that in some mice, several independent insertions occurred. We expect that independently inserted sequences will be parted by chromosomal crossover in the next generations, whereas multimers will not be. mouse N1. The presence of $h A C E 2, G F P$, the STOP cassette, and the terminator was confirmed

Breeding with Cre-mice and tamoxifen-induced activation

As an activator line, we used $\mathrm{B} 6$. Cg-Ndor $1 \mathrm{Tg}(\mathrm{UBC}-$ cre/ERT2)1Ejb/1 J, also known as Ubi-Cre, from the Jackson Laboratory (Goodwin et al., 2019). We crossed hACE2(LoxP-STOP) F0 transgenic female \# 1190 , further referred to as mouse N1, with 8.5 copies of the transgene per genome with the Ubi-Cre transgenic male \#1720 and obtained a litter consisting of 7 pups. Two of them (\# 2344, further referred to as mouse N2, and \# 2346, further referred to as mouse N3) were hACE2 (LoxP-STOP)-positive, both with 2 copies of the transgene; 3 of them (mouse N2, \# 2347, and \# 2350) were Ubi-Cre-positive, and only mouse $\mathrm{N} 2$ was positive for both hACE2 (LoxP-STOP) and Ubi-Cre (further referred to as hACE2 (LoxP-Cre)) (Fig. 3). Then, we activated Cre recombinase in this mouse N2 with tamoxifen, and GFP fluorescence was observed in its skin via a UV lamp in just one week.

Next, we measured the hACE2 (P3 and P4 primers) mRNA levels relative to the $\mathrm{mHprt}$ housekeeping gene (P11 and P12) in PBMCs by real-time PCR. We measured the $h A C E 2$ expression level in the mouse $\mathrm{N} 2$ hACE2(LoxP-Cre) after tamoxifen activation, in the mouse N3 hACE2(LoxP-STOP) with the transgene copy number, which was identical to that in mouse $\mathrm{N} 2$, and in a wild-type mouse (Fig. 4a; the hACE2 


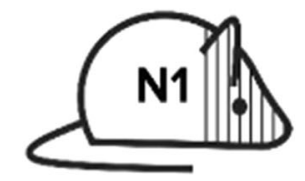

Fo oq hACE2(LOXP-Stop), mosaic
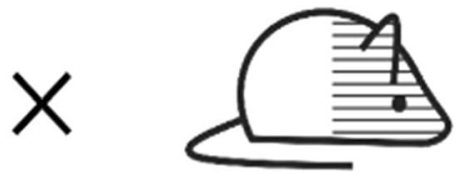

Ubi-Cre o', hemizygous

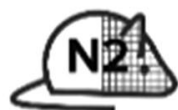

F1 O'nACE2(LOXP.Cre)

2 copies, hemizygous Ubi-Cre, hemizygous
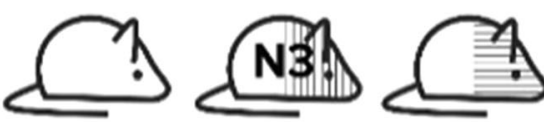

F1 o'hACE2(LOXP-Stop), Ubi-Cre,

2 copies, hemizygous hemizygous
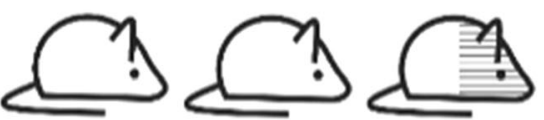

Ubi-Cre, hemizygous

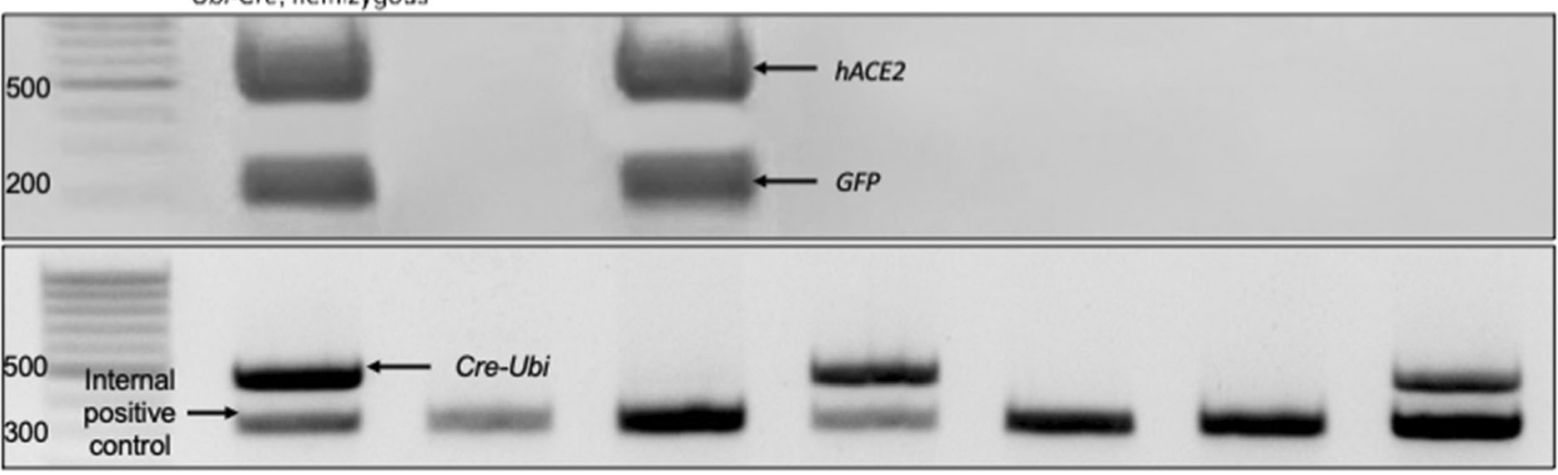

Fig. 3 Scheme of transgenic mouse breeding and F1 genotyping. Seven pups characterized by $h A C E 2$ ( $\approx 500$ bp band) and GFP ( $\approx 200 \mathrm{bp}$ band) on the upper gel and by Cre-Ubi presence ( 2 bands in the transgenic mice, 1 band in wild-type mice) on the lower gel

expression level after activation was assigned a value of 1). In mouse $\mathrm{N} 2, h A C E 2$ expression was much higher than that in the two other mice. $h A C E 2$ expression was not detected in wt mice. Transgenic mouse N3 without activation showed an expression level that was approximately $3 \%$ of that of the activated mouse N2. This probably indicated lowlevel leakage of the STOP cassette. Transgenic gDNA contamination was not the cause because the PCR product was not detectable without reverse transcription (data not shown). In mouse N2, we measured the copy numbers of $h A C E 2$ and the STOP cassette before and after activation. For this, we performed real-time PCR with the P3 and P4 primers for hACE2 and with the P13 and P14 primers for the STOP cassette using genomic DNA extracted before and after activation as the templates. We speculated that recombination would occur not only between adjacent LoxP sites but also between random sites in the case of multimeric insertion. However, we did not observe such recombination in our experiment. After tamoxifen activation, the STOP cassette copy number decreased by $30 \%$, whereas the $h A C E 2$ copy number remained exactly the same (Fig. 4b). This experiment shows that tamoxifen induction works properly and effectively, and no transgene excision takes place.

To explore transgenic hACE2 at the protein level, we performed Western blot analysis with polyclonal anti-hACE2 rabbit antibodies (Cloud-Clone Corp., USA-China). We detected a band of approximately $120 \mathrm{kDa}$ corresponding to the glycosylated hACE2 form only in the blood of the activated mouse N2. This band was not detected in the wild-type mice or in the nonactivated transgenic mouse N3 (Fig. 5). Glycosylation is an important process for membrane proteins such as ACE2. The weight of the detected band affirmed proper posttranslational modifications because the unglycosylated protein has a molecular weight of approximately 85-90 kDa (Warner 2004). 

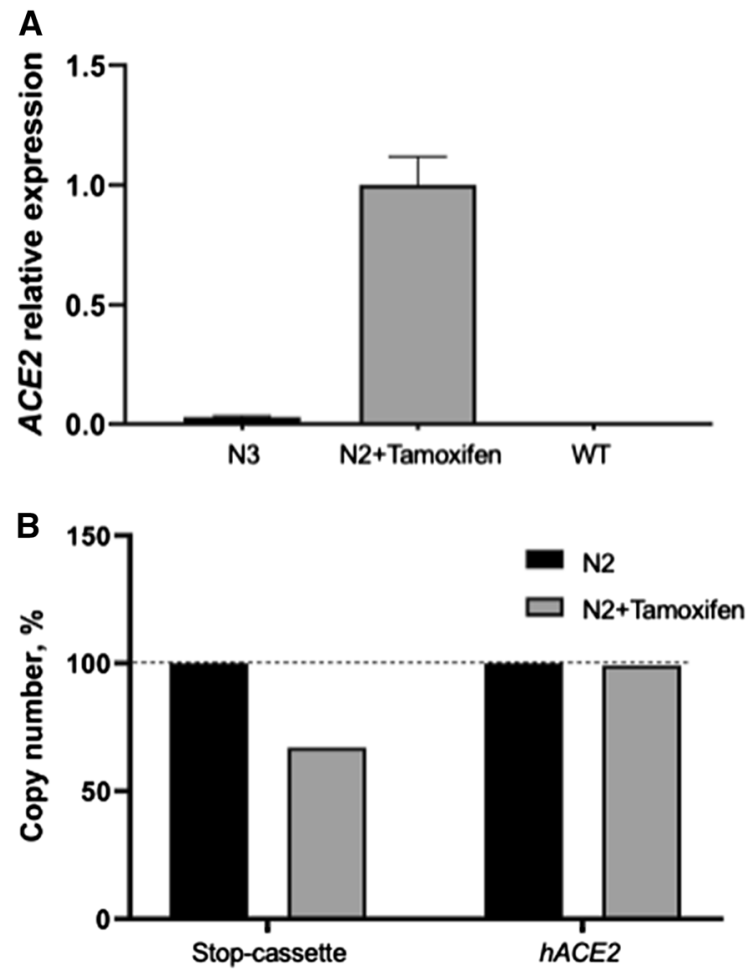

Fig. 4 Effect of tamoxifen administration on transgene activation. a hACE2 expression was found in only hACE2(LoxP-Cre) mice (N2) after tamoxifen activation. B) The Stopcassette copy number in hACE2(LoxP-Cre) mice (N2) after tamoxifen activation was decreased by $30 \%$, whereas the hACE2 copy number did not change

Finally, we measured hACE2 expression by flow cytometry with the same primary antibodies. We observed that $16 \%$ of blood mononuclei in mouse N2 were stained positively for hACE2, whereas no hACE2 + cells were detected in the nonactivated mouse N3 or in the wt mouse. As hACE2 was not detected in mouse N3 (Fig. 5) by Western blot or flow cytometry, we can conclude that there was no STOP cassette leakage at the protein level. The difference between mRNA and protein levels can be explained by the SV40polyA sequence in the $3^{\prime}$ part of the STOP cassette. Even if low-rate transcription occurs from the CAG promoter, translation of this transcript terminates at SV40polyA before the ribosome reaches the hACE2 ORF. The fact that STOP cassette leakage was observed at only the mRNA level and not at the protein level supports our claim that our animals satisfy the requirement of tamoxifen-induced expression. As we stained the cells without fixation and permeabilization, we presume that the antibodies reacted with only
hACE2 translocated to the cell surface. This is important because only translocated hACE2 can serve as an entry point for the coronavirus, and there are doubts regarding whether transgenic hACE2 can be properly processed in different types of cells (Fig. 5).

\section{Discussion}

In December 2019, Wuhan, the capital of Hubei Province in China, became the center of the outbreak of pneumonia with an unknown etiology. By January 7, 2020, Chinese scientists identified the causative infectious agent as a novel strain of coronavirus and named it SARS-CoV-2. SARS-CoV-2 causes COVID19 , a very complex disease with a wide spectrum of symptoms. At first, SARS-CoV-2 invades the epithelium of the upper and lower airways, but in the case of a severe course, it reaches the gas exchange units of the lungs and infects alveolar type II cells (Mason 2020).

Many reports show that immune cells (Mortaz et al. 2020; Liu et al. 2020; Ruan et al. 2020; Pontelli et al. 2020), vascular endothelium (Libby et al. 2020, Wichmann et al. 2020; Al-Ani et al. 2020; Varga et al. 2020; Ackermann et al. 2020; Monteil et al. 2020) and the nervous system (Iadecola et al. 2020; Song et al. 2020, preprint) can also be distinguished as important targets of SARS-CoV-2. Moreover, the list of potential targets for COVID-19 is growing. There are reports of the involvement of other organs with high expression of ACE2, such as the intestines, kidneys, and heart (Zaim et al. 2020). As ACE2 is highly expressed in tissues of the male reproductive system, there are concerns that SARS-CoV-2 can cause infertility in men (Sun 2020; Dutta et al. 2020). This suggestion is partially confirmed by cases of orchitis following the outbreak of SARS-CoV in 2002 (Xu et al. 2006) and sex hormone imbalance in male patients with COVID-19 (Ma et al. 2020). Therefore, COVID-19 has a very complex and multifaceted pathogenesis. The revelation of its pathways is an essential way in the search for effective approaches to therapy.

Herein, we describe novel transgenic mice with Cre-dependent coexpression of $\mathrm{h} A C E 2$ and GFP. This is the first transgenic strain allowing spatial and temporal control of $\mathrm{h} A C E 2$ expression. Previous lines constitutively expressed the $\mathrm{h} A C E 2$ transgene under 
A

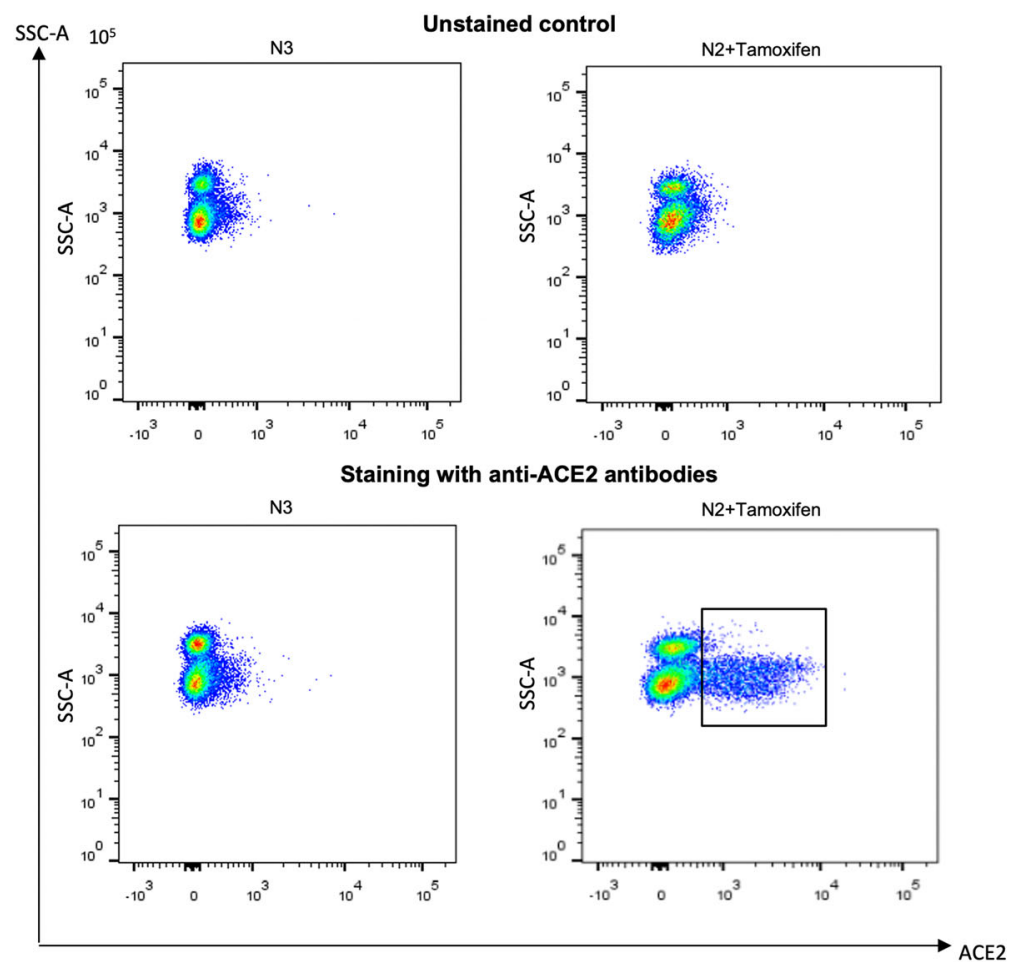

B
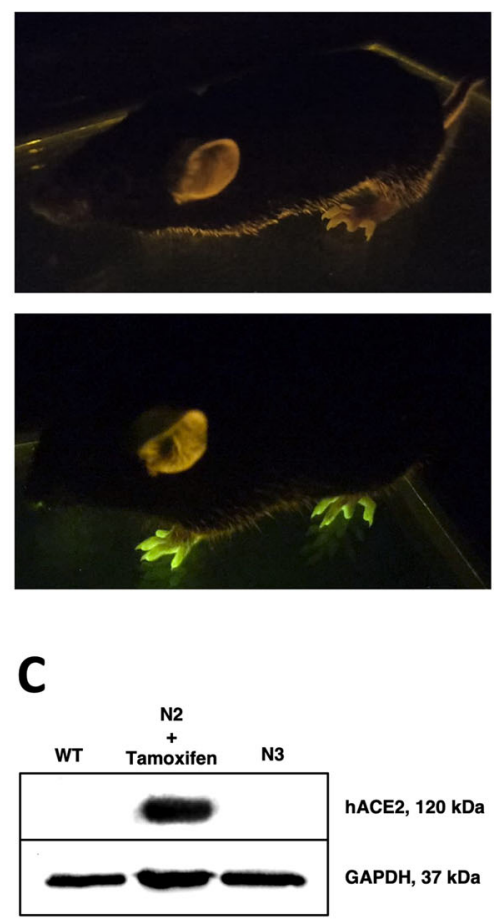

Fig. 5 Detection of hACE2 and GFP. a hACE2 presence on the surface of PBMCs. a Activated transgenic mouse N2 hACE2(LoxP-Cre) (right lower panel) compared to the nonactivated transgenic mouse N3 hACE2(LoxP-Stop) (left lower panel). Unstained controls are represented in the upper panel. b The N2 mouse skin in the UV chamber before (upper) and

the control of nonspecific promoters K18 (McCray et al. 2007) and CAG (Tseng et al. 2007) and a specific murine Ace 2 promoter (Yang et al. 2007). Although existing strains can serve for preclinical trials of antiSARS-CoV-2 agents and research on some details of virus invasion, they are not appropriate to reveal the contribution of different tissues to the pathogenesis of SARS-CoV-2 infection. The present hACE2(LoxPStop) mice were designed mostly to study the role of different tissues in the development of COVID-19 symptoms and complications. For instance, specific activation of $h A C E 2$ in endothelial, immune, or alveolar cells can help to reveal the primary causative tissue, the involvement of which leads to acute respiratory distress syndrome in COVID-19. Another option, for example, is to study the fertility of SARS$\mathrm{CoV}$-2-infected male mice with testicular-specific activation of $h A C E 2$. In this way, fertility will depend after (lower) tamoxifen induction. c Western blot analysis of hACE2 expression in PBMCs. Glycosylated $120 \mathrm{kDa}$ hACE2 in the upper panel and $37 \mathrm{kDa}$ GAPDH in the lower panel. A WT mouse is shown on the left, an activated transgenic mouse is shown in the middle, a nonactivated transgenic mouse is shown on the right

only on testicular but not on systemic and behavioral changes caused by COVID-19.

hACE2(LoxP-Stop) mice must be crossed with Cre- expression strains to initiate the transcription of transgenes. In brief, Cre strains are available transgenic animals expressing Cre recombinase, an enzyme that catalyzes site-specific recombination between two LoxP sites and, as a result, deletion of the LoxPlimited sequence in the proper orientation. Thus, if Cre recombinase is expressed, it excises the STOP cassette, resulting in the expression of hACE2 and $G F P$. In our study, we examined the $\mathrm{F} 1$ generation obtained after crossing the hACE2(LoxP-Stop) F0 mouse with the Ubi-Cre mouse. The human ubiquitin C (UBC) promoter that drives Cre expression in this strain provides a relatively consistent expression level across different cell types. One of the offspring carried both $h A C E 2 G F P$ and $C r e$ alleles. In this mouse, the 
expression of hACE2 and GFP was then successfully triggered by tamoxifen administration, at least in blood mononuclei and skin.

Choosing Ubi-Cre/ERT2 as the first strain to excise the floxed STOP cassette, we were guided by considerations of biosafety because tamoxifen-dependent activation is a two-level defense against SARS-CoV-2 infection, simplifying work with animals. Before activation, the mice were epidemiologically safe for laboratory staff, and workers were safe for the mice. Thus, it minimizes all the risks related to undesired infection of the mice by SARS-CoV-2 before placing them in specific conditions of a virological laboratory. However, other Cre activators can be chosen according to different research aims in further studies.

In this study, after activation, we detected STOP cassette excision, hACE2 mRNA presence, and hACE2 protein assembly. Western blot analysis found hACE2 with a molecular weight of approximately $120 \mathrm{kDa}$, indicating proper protein glycosylation. Using FACS, we confirmed that hACE2 is located on the cell surface, which means that transgenic protein successfully underwent surface translocation and can interact with SARS-CoV-2. Interestingly, our dosage scheme of tamoxifen administration led to relatively modest activation of the transgene, but it can be increased in further experiments if required (Donocoff et al. 2020). Thus, tamoxifen-dependent activation is an additional option to adjust transgene expression and, therefore, SARS-CoV-2 sensitivity.

Developing our strategy for the generation of mice with Cre-dependent activation of hACE2, we were guided by several points. First, we decided to coexpress hACE2 with GFP because we think it simplifies visualization procedures. GFP fluorescence confirms transgene activation, allowing visualization of hACE2-expressing tissues without the use of complicated and expensive immunostaining protocols. In our study, we demonstrated that activation of hACE2/GFP expression can be confirmed just by fluorescence in transgenic mouse skin. Notably, some authors notice that floxed transgenes can be expressed at a small level even before Cre-dependent activation (Van Hove et al., 2019), so coexpression with GFP also allows immediate detection of this leak. In our study, we did not perform the analysis of hACE2 expression before tamoxifen administration because we tried to minimize all the invasive procedures with valuable N2 transgenic mice. Nevertheless, we did not detect any green fluorescence of the skin of the nonactivated transgene in the UV chamber.

Finally, recognizing that our strain is a hybrid of CBA and C57Bl/6 J, we see that it must be returned to an inbred background for more representative research results in further studies. The most appropriate background to return to is $\mathrm{C} 57 \mathrm{Bl} / 6 \mathrm{~J}$ because almost all of the activator Cre lines are contained on this background.

\section{Conclusions}

SARS-CoV-2-sensitive murine models are an important component of studies on COVID-19. In our laboratory, a novel mouse strain that conditionally coexpresses hACE2 and GFP was obtained. We believe that our strain, hACE2(LoxP-Stop), can serve as a useful tool for studying the roles of different tissues in COVID-19 pathogenesis. Moreover, Credependent expression of hACE2 makes hACE2(LoxPStop) mice convenient for housing because before activation, they cannot serve as a reservoir for SARSCoV-2 and do not require specific biosafety conditions. Finally, coexpression with GFP simplifies the visualization of hACE2-expressing tissues. We are ready to transfer hACE2(LoxP-STOP) mice to any research group of interest. We believe that our hACE2(LoxP-STOP) strain has a number of unique properties that can be useful in preclinical trials and especially useful for studying COVID-19 pathogenesis.

Acknowledgements Authors are thankful to Alexandra Proskura (Institute for Urology and Reproductive Health, Sechenov University, Russia) for human kidney samples, to dr. Dimitry Mazurov (Institute of Gene Biology, Russian academy of sciences, Russia) for providing antibodies, to Ekaterina Varlamova (Institute of Gene Biology, Russian academy of sciences, Russia) for laboratory support, to researchers of the State Research Center of Virology and Biotechnology VECTOR for critical discussion. This work was supported by the grant 075-15-2019-1661 from the Ministry of Science and Higher Education of the Russian Federation (all animal experiments) and Institute of functional genomics of the Lomonosov Moscow State University state funding (in part related to the preparation of hACE2 cDNA). Co-workers of the Laboratory of Genome Editing for veterinary and biomedicine were supported by the Belgorod Scientific and Educational Center "Innovative Solutions in the Agro-Industrial Complex". The study was performed using the equipment of IGB RAS facilities supported by the Ministry of Science and Higher Education of the Russian Federation. 
Author contributions AD: main idea, design of the experiments, embryo transfer, obtaining the results, writing the article. DK: animal surgery, animal care, tamoxifen administration, pronuclear microinjections, tissue sampling. AB: molecular biology, DNA transgenic construcrion development, writing the article. EK: animal surgery, animal care, animal breeding, animal handling, obstetrics procedures, veterinary, blood collection. MK: molecular biology, DNA transgenic construcrion development. AK: FASC, writing the article. YuS: FASC. PS: cDNA cloning. LI: bioinformatics, mathematical proceeding. SV: main idea, design of the experiments, graphical design, writing the article.

\section{Declarations}

Conflict of interest The authors declare that there are no conflicts of interest regarding the publication of this paper.

Ethical approval Human biomaterials were obtained in the process of medical intervention under the supervision of the Local Ethics Committee of Sechenov (Medical) University. All animal experiments were carried out in accordance with the rules of humane treatment and were approved and controlled by the IGB RAS Bioethics Commission (the conclusion of the Bioethical Commission \# 06-01/02-1 dated 02/03/2020). The animal experiments did not pose any biological hazards and did not require a biosafety facility.

\section{Appendix}

See Table 1

Table 1 Sequences of primers

\begin{tabular}{ll}
\hline Code & Sequence $5^{\prime} \rightarrow 3^{\prime}$ \\
\hline P1 & AAAATTGTGTACCTTTAGCTTTTTA \\
P2 & CCTAGGAATGCTCGTCAAGA \\
P3 & CTTATGTGCACAAAGGTGAC \\
P4 & TGTGGCTGCAGAAAGTGA \\
P5 & GAGCGTTGGGCTTACCTCAC \\
P6 & GACTGCGGGTCGGCATGA \\
P7 & CCACCTCGATGACCTGCCCGG \\
P8 & CTGATGCCTCTGCTCCCCTTCC \\
P9 & GACTTCAAGACCGACCTGCG \\
P10 & TTGGGCATGATGGTGACACG \\
P11 & GCAGTACAGCCCCAAAATGG \\
P12 & CCTTTTCACCAGCAAGCT \\
P13 & GTTAGATCTGCTGCCACCGT \\
P14 & AGGTGGCAAGTGGTATTCCG \\
P15 & ATTAACCGGTATGTCAAGCTCTTCCTGGC \\
P16 & ATTAACGCGTCTAAAAGGAGGTCTGAACATCAT \\
\hline
\end{tabular}

Table 1 continued

\begin{tabular}{ll}
\hline Code & Sequence $5^{\prime} \rightarrow 3^{\prime}$ \\
\hline P17 & GACGTCACCCGTTCTGTTG \\
P18 & AGGCAAATTTTGGTGTACGG \\
P19 & CTAGGCCACAGAATTGAAAGATCT \\
P20 & GTAGGTGGAAATTCTAGCATCATCC \\
P21 & CGACGTAAACGGCCACAAGT \\
P22 & GGCGGACTTGAAGAAGTCGT \\
P23 & GCGAGTCCATGTCACTCAGG \\
P24 & GTGTTGCCCTTTGGAGCTTG \\
\hline
\end{tabular}

\section{References}

Ackermann M, Verleden SE, Kuehnel M, Haverich A, Welte T, Laenger F, Vanstapel A, Werlein C, Stark H, Tzankov A, Li WW, Li VW, Mentzer SJ, Jonigk D (2020) Pulmonary vascular endothelialitis, thrombosis, and angiogenesis in Covid-19. N Engl J Med 383(2):120-128. https://doi.org/ 10.1056/NEJMoa2015432.PMID:32437596;PMCID: PMC7412750

Al-Ani F, Chehade S, Lazo-Langner A (2020) Thrombosis risk associated with COVID-19 infection. A Scoping Rev Thromb Res 192:152-160. https://doi.org/10.1016/j. thromres.2020.05.039

Bao L, Deng W, Huang B et al (2020) The pathogenicity of SARS-CoV-2 in hACE2 transgenic mice. Nature 583:830-833. https://doi.org/10.1038/s41586-020-2312-y

Blanco-Melo D, Nilsson-Payant BE, Liu WC et al (2020) Imbalanced host response to SARS-CoV-2 drives development of COVID-19. Cell 181(5):1036-1045. https://doi. org/10.1016/j.cell.2020.04.026

Chen ZY, Yant SR, He CY, Meuse L, Shen S, Linear KMA, DN, (2001) As concatemerize in vivo and result in sustained transgene expression in mouse liver. MolTher 3:403-410

Chung JH, Whiteley M, Felsenfeld G (1993) A 5' element of the chicken beta-globin domain serves as an insulator in human erythroid cells and protects against position effect in Drosophila. Cell 74(3):505-514

Deykin A, Tikhonov M, Kalmykov V, Korobko I, Georgiev P, Maksimenko O (2019) Transcription termination sequences support the expression of transgene product secreted with milk. Transgenic Res 28(3-4):401-410. https://doi. org/10.1007/s11248-019-00122-9 (PMID: 30919251)

Dinnon KH, Leist SR, Schäfer A et al (2020) A mouse-adapted model of SARS-CoV-2 to test COVID-19 countermeasures. Nature 586:560-566

Donocoff RS, Teteloshvili N, Chung H, Shoulson R, Creusot RJ (2020) Optimization of tamoxifen-induced Cre activity and its effect on immune cell populations. Sci Rep 10(1):15244. https://doi.org/10.1038/s41598-020-72179-0

Dutta S, Sengupta P (2020) SARS-CoV-2 and Male Infertility: Possible Multifaceted Pathology. Reprod Sci 1-4. doi:https://doi.org/10.1007/s43032-020-00261-z 
Gao Q, Bao L, Mao H, Wang L, Xu K, Yang M, Li Y, Zhu L, Wang N, Lv Z, Gao H, Ge X, Kan B, Hu Y, Liu J, Cai F, Jiang D, Yin Y, Qin C, Li J, Gong X, Lou X, Shi W, Wu D, Zhang H, Zhu L, Deng W, Li Y, Lu J, Li C, Wang X, Yin W, Zhang Y, Qin C (2020) Development of an inactivated vaccine candidate for SARS-CoV-2. Science 369(6499):77-81. https://doi.org/10.1126/science. abc1932

Goodwin LO, Splinter E, Davis TL, Urban R, He H, Braun RE, Chesler EJ, Kumar V, van Min M, Ndukum J, Philip VM, Reinholdt LG, Svenson K, White JK, Sasner M, Lutz C, Murray SA (2019) Large-scale discovery of mouse transgenic integration sites reveals frequent structural variation and insertional mutagenesis. Genome Res 29(3):494-505. https://doi.org/10.1101/gr.233866.117

$\mathrm{Gu} \mathrm{H}$ et al (2020) Adaptation of SARS-CoV-2 in BALB/c mice for testing vaccine efficacy. Science 369:1603-1607

Hassan AO et al (2020) A SARS-CoV-2 infection model in mice demonstrates protection by neutralizing antibodies. Cell 182:744-753

Hoffmann M, Kleine-Weber H, Schroeder S et al (2020) SARSCoV-2 cell entry depends on ACE2 and TMPRSS2 and Is blocked by a clinically proven protease inhibitor. Cell 181(2):271-280.e8. https://doi.org/10.1016/j.cell.2020.02. 052

Iadecola C, Anrather J, Kamel H (2020) Effects of COVID-19 on the nervous system. Cell 183(1):16-27.e1. https://doi. org/10.1016/j.cell.2020.08.028

Lakdawala SS, Menachery VD (2020) The search for a COVID19 animal model. Science 368(6494):942-943. https://doi. org/10.1126/science.abc6141

Lakso M, Sauer B, Mosinger B Jr et al (1992) Targeted oncogene activation by site-specific recombination in transgenic mice. ProcNatlAcadSci USA 89(14):6232-6236. https://doi.org/10.1073/pnas.89.14.6232

Libby P, Lüscher T (2020) COVID-19 is, in the end, an endothelial disease. Eur Heart J 41(32):3038-3044. https:// doi.org/10.1093/eurheartj/ehaa623.PMID: 32882706;PMCID:PMC7470753

Liu J, Li S, Liu J, Liang B, Wang X, Wang H et al (2020) Longitudinal characteristics of lymphocyte responses and cytokine profiles in the peripheral blood of SARS-CoV-2 infected patients. EBioMedicine 55:102763. https://doi. org/10.1016/j.ebiom.2020.102763

Lu R, Zhao X, Li J, Niu P, Yang B, Wu H (2020) Genomic characterisation and epidemiology of 2019 novel coronavirus: implications for virus origins and receptor binding. Lancet 395:565-574

Ma L, Xie W, Li D, Shi L, Mao Y, Xiong Y, et al (2020) Effect of SARS-CoV-2 infection upon male gonadal function: a single center-based study. medRxiv. https://doi.org/10. 1101/2020.03.21.20037267

Masemann D, Ludwig S, Boergeling Y (2020) Advances in transgenic mouse models to study infections by human pathogenic viruses. Int J MolSci 21:9289

Mason RJ (2020) Pathogenesis of COVID-19 from a cell biology perspective. EurRespir J 55(4):2000607. https://doi. org/10.1183/13993003.00607-2020

McCray PB Jr, Pewe L, Wohlford-Lenane C et al (2007) J Virol 81(2):813-821. https://doi.org/10.1128/JVI.02012-06
Meirson T, Bomze D, Markel G (2020) Structural basis of SARS-CoV-2 spike protein induced by ACE2. Bioinformatics 20:744. https://doi.org/10.1093/bioinformatics/ btaa744

Menachery VD et al (2016) SARS-like WIV1-CoV poised for human emergence. ProcNatlAcadSci 113:3048-3053

Miyazaki J, Takaki S, Araki K, Tashiro F, Tominaga A, Takatsu K, Yamamura K (1989) Expression vector system based on the chicken beta-actin promoter directs efficient production of interleukin-5. Gene 79(2):269-277. https://doi.org/10. 1016/0378-1119(89)90209-6 (PMID: 2551778)

Monteil VKH, Prado P, Hagelkrüys A et al (2020) Inhibition of SARS-CoV-2 infections in engineered human tissues using clinical-grade soluble human ACE2. Cell. https://doi.org/ 10.1016/j.cell.2020.04.004

Mortaz E, Tabarsi P, Varahram M, Folkerts G, Adcock IM (2020) The immune response and immunopathology of COVID-19. Front Immunol 11:2037. https://doi.org/10. 3389/fimmu.2020.02037

Pontelli MC et al (2020) Infection of human lymphomononuclear cells by SARS-CoV-2. Biorxiv. https://doi.org/10. 1101/2020.07.28.225912

Ruan Q, Yan K, Wang W, Jiang L, Song J (2020) Clinical predictors of mortality due to COVID-19 based on an analysis of data of 150 patients from Wuhan, China. Intens Care Med 46:846-848. https://doi.org/10.1007/s00134020-05991-X

Shang J, Ye G, Shi K et al (2020) Structural basis of receptor recognition by SARS-CoV-2. Nature 581:221-224. https:// doi.org/10.1038/s41586-020-2179-y

Silaeva YY, Kalinina AA, Vagida MS et al (2013) Decrease in pool of $\mathrm{T}$ lymphocytes with surface phenotypes of effector and central memory cells under Influence of TCR transgenic $\beta$-chain expression. Biochem Moscow 78:549-559. https://doi.org/10.1134/S0006297913050143

Silaeva YY, Kirikovich YK, Skuratovskaya LN et al (2018) Optimal number of embryos for transplantation in obtaining genetic-modified mice and goats. Russ J Dev Biol 49:356-361. https://doi.org/10.1134/ S106236041806005X

Smirnov A, Fishman V, Yunusova A et al (2020) DNA barcoding reveals that injected transgenes are predominantly processed by homologous recombination in mouse zygote. Nucleic Acids Res 48(2):719-735. https://doi.org/10.1093/ nar/gkz1085

Soldatov VO, Kubekina MV, Silaeva YuYu, Bruter AV, Deykin AV (2020) On the way from SARS-CoV-sensitive mice to murine COVID-19 model. Res Results Pharmacol 6(2):1-7. https://doi.org/10.3897/rrpharmacology.6.53633

Song E, Zhang C, Israelow B, Lu P, Weizman O-E, Liu F, Dai Y, Szigeti-Buck K, Yasumoto Y, Wang G et al (2020) Neuroinvasive potential of SARS-CoV-2 revealed in a human brain organoid model. BioRxiv. https://doi.org/10.1101/ 2020.06.25.169946

Subbarao K, McAuliffe J, Vogel L et al (2004) Prior infection and passive transfer of neutralizing antibody prevent replication of severe acute respiratory syndrome coronavirus in the respiratory tract of mice. J Virol 78(7):3572-3577

Sun J (2020) The hypothesis that SARS-CoV-2 affects male reproductive ability by regulating autophagy. Med 
Hypotheses 143:110083. https://doi.org/10.1016/j.mehy. 2020.110083

Sun J et al (2020) Generation of a broadly useful model for COVID-19 pathogenesis, vaccination, and treatment. Cell 182:734-743

Suzuki T (2020) Generation of human bronchial organoids for SARS-CoV-2 research. BioRxiv. https://doi.org/10.1101/ 2020.05.25.115600

Tseng CT et al (2007) Severe acute respiratory syndrome coronavirus infection of mice transgenic for the human Angiotensin-converting enzyme 2 virus receptor. J Virol 81:1162-1173

Van Hove H, Antunes ARP, De Vlaminck K, Scheyltjens I, Van Ginderachter JA, Movahedi K (2020) Identifying the variables that drive tamoxifen-independent CreERT2 recombination: implications for microglial fate mapping and gene deletions. Eur J Immunol 50(3):459-463. https:// doi.org/10.1002/eji.201948162 (PMID: 31785096)

Varga Z, Flammer AJ, Steiger P, Haberecker M, Andermatt R, Zinkernagel AS, Mehra MR, Schuepbach RA, Ruschitzka F, Moch H (2020) Endothelial cell infection and endotheliitis in COVID-19. Lancet 395(10234):1417-1418. https://doi.org/10.1016/S0140-6736(20)30937-5.PMID: 32325026;PMCID:PMC7172722

Warner FJ, Smith AI, Hooper NM, Turner AJ (2004) Angiotensin-converting enzyme-2: a molecular and cellular perspective. Cell Mol Life Sci 61(21):2704-2713. https:// doi.org/10.1007/s00018-004-4240-7.PMID: 15549171;PMCID:PMC7079784

Wichmann D, Sperhake JP, Lütgehetmann M et al (2020) autopsy findings and venous thromboembolism in patients with COVID-19: A prospective cohort study. Ann Intern Med 173(4):268-277. https://doi.org/10.7326/M20-2003

Xu J, Qi L, Chi X, Yang J, Wei X, Gong E, Peh S, Gu J (2006) Orchitis: a complication of severe acute respiratory syndrome (SARS). BiolReprod 74(2):410-416. https://doi. org/10.1095/biolreprod.105.044776

Yang XH et al (2007) Mice transgenic for human angiotensinconverting enzyme 2 provide a model for SARS coronavirus infection. Comp Med 57:450-459

Zaim S, Chong JH, Sankaranarayanan V, Harky A (2020) COVID-19 and multiorgan response. CurrProblCardiol 45(8):100618. https://doi.org/10.1016/j.cpcardiol.2020. 100618

Zhu N (2019) A novel coronavirus from patients with pneumonia in China. N Engl J Med 382:727-733

Zvartsev RV, Korshunova DS, Gorshkova EA, Nosenko MA, Korneev KV, Maksimenko OG, Korobko IV, Kuprash DV, Drutskaya MS, Nedospasov SA, Deikin AV (2019) Neonatal lethality and inflammatory phenotype of the new transgenic mice with overexpression of human interleukin6 in myeloid cells. Dokl Biochem Biophys. 483(1):344-347. https://doi.org/10.1134/ S1607672918060157 (PMID: 30607736)

Zvezdova ES, Silaeva YY, Vagida MS et al (2010) Generation of transgenic animals expressing the $\alpha$ and $\beta$ chains of the autoreactive T-cell receptor. MolBiol 44:277-286. https:// doi.org/10.1134/S0026893310020135

Publisher's Note Springer Nature remains neutral with regard to jurisdictional claims in published maps and institutional affiliations. 